\title{
Analytical and Numerical Results for an Adhesively Bonded Joint Subjected to Pure Bending
}

\author{
Stanley S. Smeltzer III ${ }^{*}$ \\ 8 W. Taylor St., NASA Langley Research Center, Hampton, VA, 23681-2199 \\ and \\ Eric Lundgren ${ }^{\dagger}$ \\ San Diego State University, San Diego, CA, 92182
}

\begin{abstract}
A one-dimensional, semi-analytical methodology that was previously developed for evaluating adhesively bonded joints composed of anisotropic adherends and adhesives that exhibit inelastic material behavior is further verified in the present paper. A summary of the first-order differential equations and applied joint loading used to determine the adhesive response from the methodology are also presented. The method was previously verified against a variety of single-lap joint configurations from the literature that subjected the joints to cases of axial tension and pure bending. Using the same joint configuration and applied bending load presented in a study by Yang, the finite element analysis software ABAQUS was used to further verify the semi-analytical method. Linear static ABAQUS results are presented for two models, one with a coarse and one with a fine element meshing, that were used to verify convergence of the finite element analyses. Close agreement between the finite element results and the semi-analytical methodology were determined for both the shear and normal stress responses of the adhesive bondline. Thus, the semi-analytical methodology was successfully verified using the ABAQUS finite element software and a single-lap joint configuration subjected to pure bending.
\end{abstract}

\section{Nomenclature}

$\begin{array}{ll}\boldsymbol{A}_{\mathrm{ij}} & =\text { extensional stiffness matrix } \\ \boldsymbol{B}_{\mathrm{ij}} & =\text { bending-extensional coupling stiffness matrix } \\ \boldsymbol{D}_{\mathrm{ij}} & =\text { bending stiffness matrix } \\ \boldsymbol{E}_{s} & =\text { secant modulus of the adhesive } \\ \boldsymbol{h}_{\mathbf{L}} & =\text { thickness of the lower adherend } \\ \boldsymbol{h}_{U} & =\text { thickness of the upper adherend } \\ \mathbf{K}_{\mathrm{s}} & =\text { shear correction factor } \\ \mathbf{Q}_{\boldsymbol{x}} & =\text { transverse force resultant } \\ \mathbf{M}_{x x} & =\text { bending and twisting moment resultant } \\ \mathbf{N}_{x x} & =\text { membrane stress resultant }\end{array}$

\footnotetext{
* Structures Research Engineer, Mechanics of Structures and Materials Branch. Senior Member, AIAA and Member, ASME.

${ }^{\dagger}$ Student, Mechanical and Aerospace Department. Member, AIAA and ASME.
} 


$\begin{array}{ll}t & =\text { thickness of the adhesive } \\ (\mathrm{x}, \mathrm{y}, \mathrm{z}) & =\text { reference coordinate system } \\ \mu_{p} & =\text { plastic Poisson's ratio of the adhesive } \\ \varepsilon_{z z} & =\text { normal strain component in the } \mathrm{z} \text { direction } \\ \gamma_{x z} & =\text { engineering shear strain component } \\ \sigma_{z z} & =\text { normal stress component in the } \mathrm{z} \text { direction } \\ \tau_{x z} & =\text { shear stress component } \\ ()_{, x} & =\text { derivative with respect to } x\end{array}$

\section{Introduction}

$\mathbf{R}$ apid -solution analysis methods that are capable of predicting the stress response of bonded joints in Raircraft, spacecraft, and launch vehicle structures represent a key area of technology development for design engineers. Presently, joint designs are primarily determined by using either finite element methods (FEM) or simple special purpose codes (e.g., one- or two-dimensional analytical methods) in conjunction with validation testing. The special purpose codes are generally efficient, user-intensive, and lend themselves to conducting parametric studies; however, they are limited to one- or two-dimensional analyses of specific joint configurations. Conversely, FEM are capable of evaluating joints with complex geometry and loading, but are very inefficient for conducting design studies and have serious problems with convergence of analysis results. The proposed paper is intended to provide further verification of a Langley-developed analysis methodology ${ }^{1,2}$, which provides efficient tailoring of various joint configurations while incorporating features such as anisotropic adherend behavior, inelastic adhesive behavior, and first-order shear deformation.

A few different semi-analytical approaches to determining the stress response in adhesively bonded joints have been developed in the past few years, primarily involving the use of classical laminated plate theory. Yang et al. ${ }^{3}$ formulated a method using classical laminated plate theory with first-order shear deformation to analyze symmetric and asymmetric single-lap joints subjected to tensile and bending loading. Mortensen and Thomsen ${ }^{4}$ developed a general, two-dimensional analysis methodology that modeled the adherends as generally orthotropic laminates using classical laminate theory and the adhesive as both linearly elastic and inelastic. A more recent approach by Oterkus et al. ${ }^{5,6}$ determined the geometrically nonlinear response of composite single-lap and tubular bonded joints with tapered adherend edges subjected to uniaxial tension. Their method utilizes the principle of virtual work in conjunction with von Karman's nonlinear plate theory to model the adherends and a shear lag model to represent the kinematics of the thin adhesive layer between the adherends. The current emphasis to develop stiffness tailored aircraft, spacecraft, and launch vehicle structures requires a rapid-solution analysis tool that includes anisotropy for the adherends and inelastic adhesive behavior to identify a variety of preliminary bonded joint configurations.

The objective of the present paper is to summarize the semi-analytical methodology for evaluating general, adhesively bonded joint overlaps, and present results from the method along with those from the literature and a commercial FEM program for a single-lap joint subjected to pure bending. In the remainder of the paper, a summary of the analytical methodology, a description of the finite element analysis models, and a discussion of the results are given.

\section{Analytical Methodology}

A description of the basic terminology, geometry, and the internal loading and stresses for a differential element of a joint overlap that is contained within a general adhesively bonded joint is shown in Figure 1. The adhesively bonded single-lap joint is composed of two laminated composite plates, referred to as upper and lower adherends, and an adhesive layer. The adherends are assumed to behave as linear elastic, cylindrically bent plates under a plane strain condition while the adhesive layer is modeled as an isotropic, inelastic material. Specifically, components of shear and normal stress within the adhesive layer are nonlinear functions of the adhesive strains. Additionally, the effects of transverse shear deformation in the adherends are included by using first-order laminated plate theory. As a result of the assumption of cylindrical bending, only a cross-section of the entire joint is modeled; therefore, the loading in the figure is given in terms of a unit width joint. Superscript letters, $U$ for the upper adherend and $L$ for the lower 
adherend, identify variables and loading for each adherend while equivalent terms for the adhesive layer do not have any special notation.

The development of the semi-analytical field equations for the Langley-developed method, hereafter referred to as ABJO (Anisotropic Bonded Joint Optimizer), was provided in detail in previous publications $^{1,2}$. In summary, equations relating the adherend stress and moment resultants to the adhesive stress components were obtained from force and moment equilibrium, joint kinematics, constitutive relations, and inelastic yielding relationships. Specifically describing the derivation of the equilibrium equations, a section-cut of the general joint overlap was made as shown in Figure 2a to obtain the joint equilibrium equations with the desired quantities. The applied loads on the ends of the overlap region are given by $\mathbf{N}_{\boldsymbol{o}}, \mathbf{M}_{\boldsymbol{o}}$, and $\mathbf{Q}_{\boldsymbol{o}}$, while $\mathbf{Q}_{\boldsymbol{x}}, \mathbf{N}_{\boldsymbol{x} x}$, and $\mathbf{M}_{\boldsymbol{x} x}$ represent the force, stress, and moment resultants, respectively. The positive directions for the externally applied loading and internal force, stress, and moment quantities are defined in Figure 2b. Thus, a set of six field equations corresponding to a bonded single-lap joint configuration are given in equations (1)-(6).

In the adhesive layer, the normal component of the linear strain tensor is denoted by $\varepsilon_{z z}$, and $\gamma_{x z}$ denotes the engineering shear strain. The laminate thicknesses for the upper and lower adherends are given by $\boldsymbol{h}_{\boldsymbol{U}}$ and $\boldsymbol{h}_{\boldsymbol{L}}$, respectively, with $\boldsymbol{t}$ denoting the adhesive thickness. The terms $\boldsymbol{E}_{\boldsymbol{s}}$ and $\boldsymbol{\mu}_{p}$ denote the inelastic adhesive material properties, and $\mathbf{K}_{\mathrm{s}}$ is a shear correction factor.

$$
\begin{gathered}
\mathbf{N}_{x x}^{U},_{x}=\left[\frac{E_{s}}{2\left(1+\mu_{p}\right)}\right] \cdot \gamma_{x z} \\
\mathbf{Q}_{x}^{U},_{x}=\left[\frac{E_{s}}{\left(1-\mu_{p}^{2}\right)}\right] \cdot \varepsilon_{x z} \\
\mathbf{M}_{x x}^{U},_{x}=\mathbf{Q}_{x}^{U}-\left[\frac{E_{s}\left(h_{U}+t\right)}{4\left(1+\mu_{p}\right)}\right] \cdot \gamma_{x z} \\
\frac{d F}{d x}=\frac{1}{t}\left\{\frac{A^{L}}{D^{L}}\left[\mathbf{M}_{o}^{U}+\mathbf{M}_{o}^{L}-\mathbf{M}_{x x}^{U}+x\left(\mathbf{Q}_{o}^{U}+\mathbf{Q}_{o}^{L}\right)-h^{*}\left(\mathbf{N}_{x x}^{U}-\mathbf{N}_{o}^{U}\right)\right]-\frac{A^{U}}{D^{U}} \mathbf{M}_{x x}^{U}\right\}
\end{gathered}
$$

and

$$
\begin{gathered}
\frac{d \varepsilon_{z z}}{d x}=F \\
\gamma_{x z},_{x}=\frac{1}{t}\left\{\mathbf{N}_{x x}^{U}\left[\left(A_{11}^{U}\right)^{-1}+\left(A_{11}^{L}\right)^{-1}+\frac{h^{*} h_{L}}{2}\left(D_{11}^{L}\right)^{-1}\right]-\left(A_{11}^{L}\right)^{-1}\left(\mathbf{N}_{o}^{U}+\mathbf{N}_{o}^{L}\right)\right. \\
-\mathbf{M}_{x x}^{U}\left[\frac{h_{U}}{2}\left(D_{11}^{U}\right)^{-1}-\frac{h_{L}}{2}\left(D_{11}^{L}\right)^{-1}\right] \\
+\varepsilon_{z z}\left[\frac{E_{s}}{\left(1-\mu_{p}^{2}\right)}\right]\left[\left(\frac{h_{U}}{2 K_{s}^{U} A_{55}^{U}}\right)-\left(\frac{h_{L}}{2 \mathbf{K}_{s}^{L} A_{55}^{L}}\right)\right] \\
\left.-\frac{h_{L}}{2}\left(D_{11}^{L}\right)^{-1}\left[x\left(\mathbf{Q}_{o}^{U}+\mathbf{Q}_{o}^{L}\right)+\mathbf{M}_{o}^{U}+\mathbf{M}_{o}^{L}+h^{*} \mathbf{N}_{o}^{U}\right]\right\}
\end{gathered}
$$

Additionally, material properties for the adherends are defined by $A=A_{11} A_{66}-A_{16}^{2}$, $B=B_{11} A_{66}-B_{16} A_{16}, C=A_{11} B_{16}-A_{16} B_{11}$, and $D=A D_{11}-B B_{11}-C B_{16}$ where $A_{1 j}$ are the extensional stiffnesses, $\boldsymbol{B}_{\mathrm{ij}}$ are the bending-extensional coupling stiffnesses, and $\boldsymbol{D}_{\mathrm{ij}}$ are the bending stiffnesses from 
classical laminated plate theory. Thus, the system of first-order differential equations, equations (1) - (6), are used along with a total plasticity theory and a modified von-Mises yield criterion to determine the force and moment resultants and the one-dimensional strain field in the adhesive layer as a function of the joint length. The stress response for the adhesive layer is subsequently determined using the previously calculated adhesive strain field and the following constitutive equations:

$$
\begin{gathered}
\sigma_{z z}=\frac{E_{s} \varepsilon_{z z}}{\left(1-\mu_{p}^{2}\right)} \\
\tau_{x z}=\frac{E_{s} \gamma_{x z}}{2\left(1+\mu_{p}\right)}
\end{gathered}
$$

where $\sigma_{z z}$ and $\tau_{x z}$ denote the normal and shear stress in the adhesive layer, respectively.

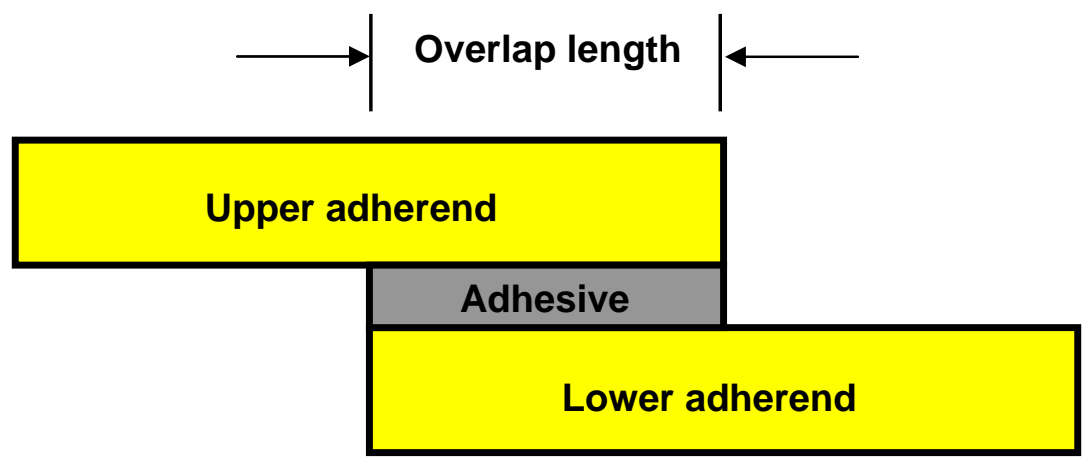

a)

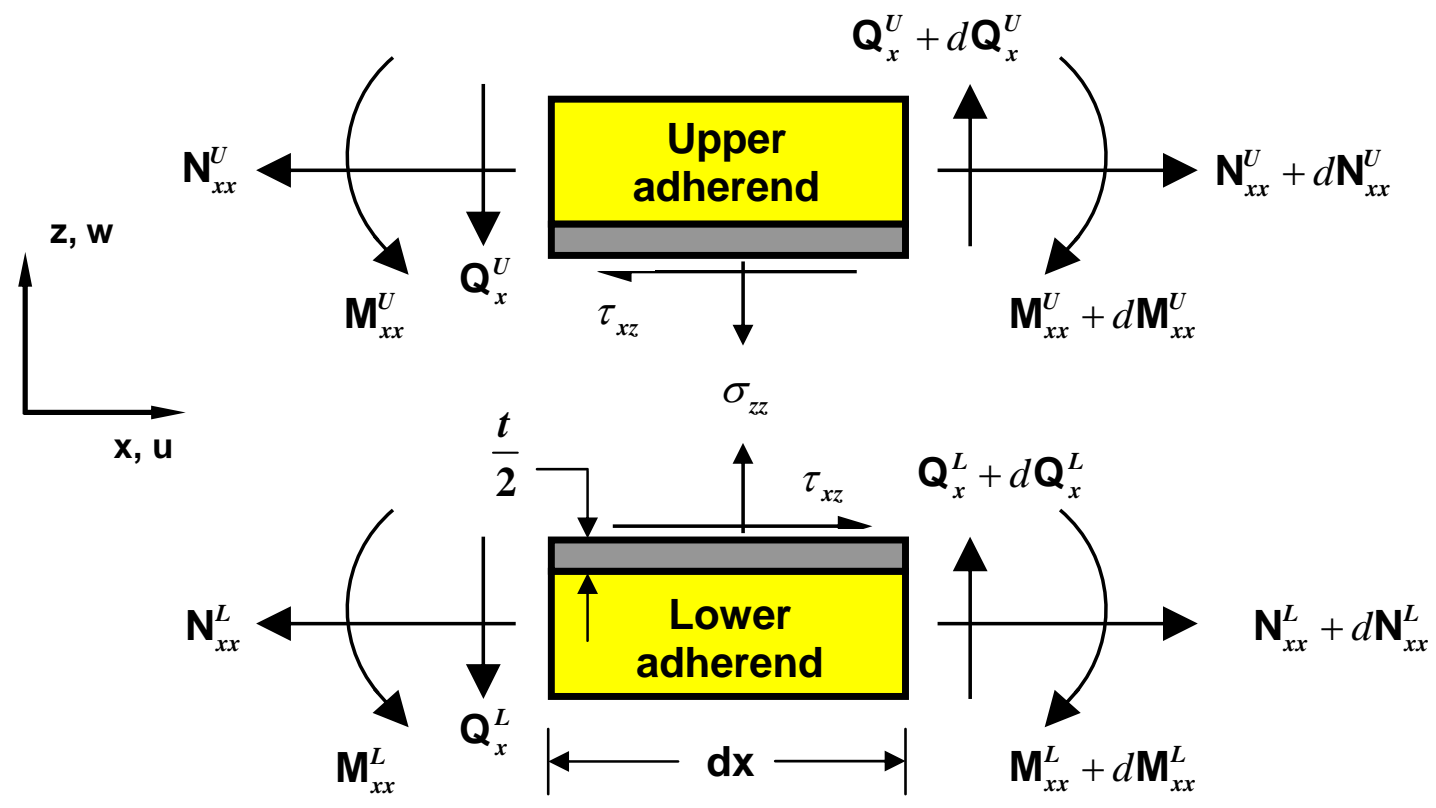

b)

Figure 1. Definitions of the a) basic terminology for an adhesively bonded single-lap joint and $b$ ) the internal adherend loading and adhesive stresses for a differential element. 


\section{Numerical Model Description}

A basic description of the applied loading and geometry for the adhesively bonded single-lap joint configuration evaluated in the present paper is shown in Figure 3. The joint configuration in Figure 3 was originally evaluated by Yang ${ }^{3}$ and used by the first author to verify the ABJO code in Reference 1 . The adherends are modeled as orthotropic materials and are defined using average laminate properties, while the adhesive layer is modeled as a linear-elastic, isotropic material. The material properties for the adherends and adhesive are given in Table 1 along with laminate configuration for the upper and lower adherends. In the present paper, the effects of adhesive yielding on the strain response of the joint were not investigated; therefore, the inelastic adhesive material properties, $\boldsymbol{\mu}_{p}$ and $\boldsymbol{E}_{\boldsymbol{s}}$, are equivalent to the Poisson's ratio and Young's modulus of the adhesive, respectively.

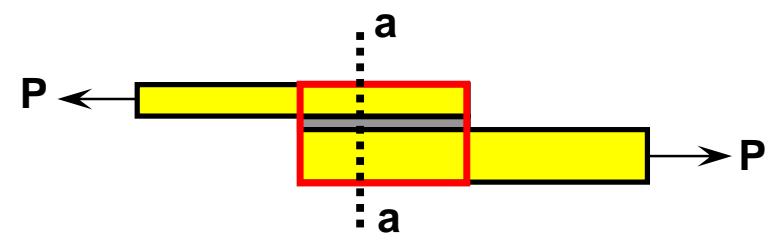

a)

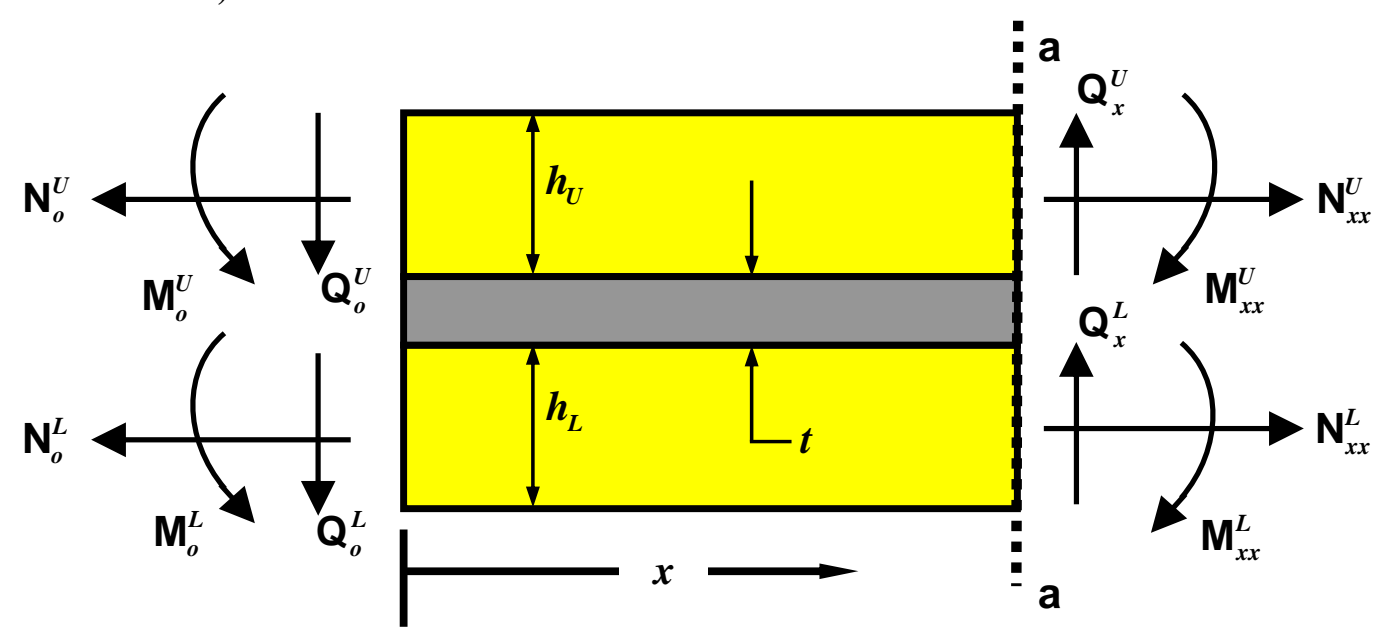

b)

Figure 2. A bonded single-lap joint subjected to axial tension depicted with a) a cut through the joint cross-section and $b$ ) the definitions of positive directions for the applied edge-joint loading and the force, stress, and moment resultants.

The longitudinal joint cross-section was evaluated in both models using the two dimensional ABAQUS CPE4I elements. The CPE4I elements are four-node, bilinear plane strain elements that have displacement compatability ( $\mathrm{C}_{0}$-continuous) and are enhanced by incompatible modes to improve their response to bending loads. In addition to standard displacement degrees of freedom, incompatible deformation modes are incorporated into the element formulation, which function to reduce the element stiffness in bending. Essentially, the incompatible mode formulation accounts for the shear locking or parasitic shear that occurs in fully integrated, bilinear four-node elements with high aspect ratio $(\mathrm{a} / \mathrm{b})$ elements.

Two models were created using the ABAQUS finite element code $^{7}$ and the single-lap joint configuration shown in Figure 3 to perform plane strain analyses of an adhesively bonded single-lap joint, and obtain results that could be correlated with results from the Langley-developed code ABJO. The fournode, bilinear plane strain elements were used to discretize both the adherend and adhesive layer in each model with the only difference between the two models being the amount of discretization or mesh density used to define the adhesive layer. Henceforth, the models will be referred to as coarse and fine for 
identification purposes, where the coarse model has half as many elements through the thickness of the adhesive layer as the fine model.

A description of the coarse model, which was composed of 6104 nodes and 5830 elements, is presented in what follows. The adhesive layer was modeled using four elements through the thickness of the adhesive layer and the node distribution was biased toward the ends of the overlap region, such that the edge elements have a unit aspect ratio. A view of an entire model and magnified views of a region of the bondline for both the coarse and fine models is shown in Figure 4. The biasing of elements within the bondline was done in an attempt to capture the singularity, i.e. peak stresses, at the adhesive free edge. The element aspect ratios increase toward the middle of the joint overlap in an attempt to reduce the computational effort for each analysis run. In both models, the adherends extend $25 \mathrm{~mm}$ beyond the overlap on both ends of the joint to ensure that the appropriate far-field boundary conditions were applied to the ends of the laminates.

The fine mesh model was the second model evaluated, and consisted of 68651 nodes and 67120 elements. The primary purpose of the fine model was to verify convergence of the adhesive strain field based on the coarse model results. As previously stated, the mesh density for the fine model was only increased in the region of interest; i.e. the adhesive bondline. Specifically, the element density through the thickness of the adhesive layer was increased by a factor of two over the coarse model, while a unit element aspect ratio was again maintained at the free edges of the adhesive overlap. Thus, the fine model used a total of eight elements in the thickness direction to model the bondline. The applied external loads, material properties, and boundary constraints were the same as those used in the coarse model.

The applied external loads and boundary constraints are given in Figure 5, and were used to simulate a case of pure bending for the single-lap joint. A force couple of $0.667 \mathrm{~N}$ was applied at the ends of the upper and lower surfaces (+/- 1.5mm) for each adherend to simulate an applied bending moment of 2 $\mathrm{N} / \mathrm{mm}^{2}$. The lower adherend was constrained at the centerline of the laminate using a pinned condition, while the upper adherend was also restrained at the laminate centerline by permitting translations in the $\mathrm{x}$ direction and rotations about the $\mathrm{z}$ axis for a total of two degrees of freedom.

Table 1. Material properties for the components of the single-lap joint model (from Yang ${ }^{3}$ ) used to compare ABJO with the ABAQUS analyses.

\begin{tabular}{c|ccc}
\hline \hline $\begin{array}{c}\text { Lamina } \\
\text { property }\end{array}$ & $\begin{array}{c}\text { Upper } \\
\text { adherend }\end{array}$ & $\begin{array}{c}\text { Lower } \\
\text { adherend }\end{array}$ & Adhesive \\
\hline $\mathrm{E}_{11},\left(\mathrm{~N} / \mathrm{mm}^{2}\right)$ & 181,000 & 181,000 & 960 \\
$\mathrm{E}_{22},\left(\mathrm{~N} / \mathrm{mm}^{2}\right)$ & 10,300 & 10,300 & 960 \\
$v_{12}$ & 0.28 & 0.28 & 0.4 \\
$\mathrm{G}_{12},\left(\mathrm{~N} / \mathrm{mm}^{2}\right)$ & 7,170 & 7,170 & - \\
$\begin{array}{c}\text { Laminate stacking } \\
\text { sequence (degrees) }\end{array}$ & {$\left[0,90_{2}, 0,90,0\right]_{\mathrm{s}}$} & {$\left[0,90_{2}, 0,90,0\right]_{\mathrm{s}}$} & - \\
\hline \hline
\end{tabular}

${ }^{\mathrm{a}}$ Subscripts 1 and 2 denote the longitudinal (fiber) and transverse (matrix) directions of an anisotropic lamina, respectively.

\section{Results}

A plot of the deformed shape from a linear static ABAQUS analysis is given in Figure 6 for the singlelap joint configuration subjected to an applied $2 \mathrm{~N}-\mathrm{mm} / \mathrm{mm}$ moment loading, as previously described in Figure 3. While the deformed shape for the single-lap joint configuration is representative of both the coarse and fine meshed models, details of the bondline deformation in the area near the end of the overlap 
region are shown in Figure 6 for both mesh density models. The deformed shape of the single-lap joint model could not be compared with deformations from ABJO, as ABJO primarily determines stresses and strains in the adhesive layer. However, the deformed shape from ABAQUS served as an additional verification that the correct loading was applied to both the coarse and fine mesh models.

The shear and normal stress responses for the adhesive layer in the single-lap joint configuration of Figure 3 was determined using ABJO and is shown in Figures 7 and 8 along with results determined by Yang. The two analysis methods had very close agreement with less than a $1 \%$ difference between the peak shear and normal adhesive stresses determined using ABJO and Yang's method. The peak shear and normal stresses determined using ABJO were $0.15 \mathrm{~N} / \mathrm{mm}^{2}$ and $0.321 \mathrm{~N} / \mathrm{mm}^{2}$, respectively. Specifically, the shear stress solutions are almost identical while a small difference in the normal stress response for the two methods is evident at the points on the joint overlap just before the ends of the joint. This difference appears to be a result of localized, nonlinear effects that make the solution sensitive near the joint ends due to large gradients in the normal stress field in those regions. The large gradients are a result of the bending deformation that occurs in the joint overlap adjacent to the ends of the joint and the free edge. Specifically, the bending occurs as the applied load is gradually increased and the adherends outside the overlap region are subjected to greater bending deformation than the stiffer overlap region. However, the difference between the two analysis methods is not a significant factor in the stress response of the adhesive bondline, since the normal stress at that location is not critical compared to the global adhesive normal stress field.

The derivation of the field equations for ABJO assumes that the strains are determined at the centerline of the adhesive layer, and are constant though the adhesive thickness. In the ABAQUS models, the discrete definition of the adhesive layer or bondline permitted the stress response to be determined in elements through the thickness direction of the adhesive layer. The results determined using ABAQUS were extracted with the ABAQUS Stress Linearization feature, which permited nodal stress values to be extracted along a user-defined line on the undeformed model. A tabular output file was generated, containing component stresses taken at a user-specified number of evenly spaced sample points along the adhesive overlap. Extraction of data points was performed for both the normal and shear stress responses of the adhesive layer. Additionally, invoking the Stress Linearization feature permitted the determination of stress responses at equally spaced points, while the mesh density was biased in favor of the joint step regions.

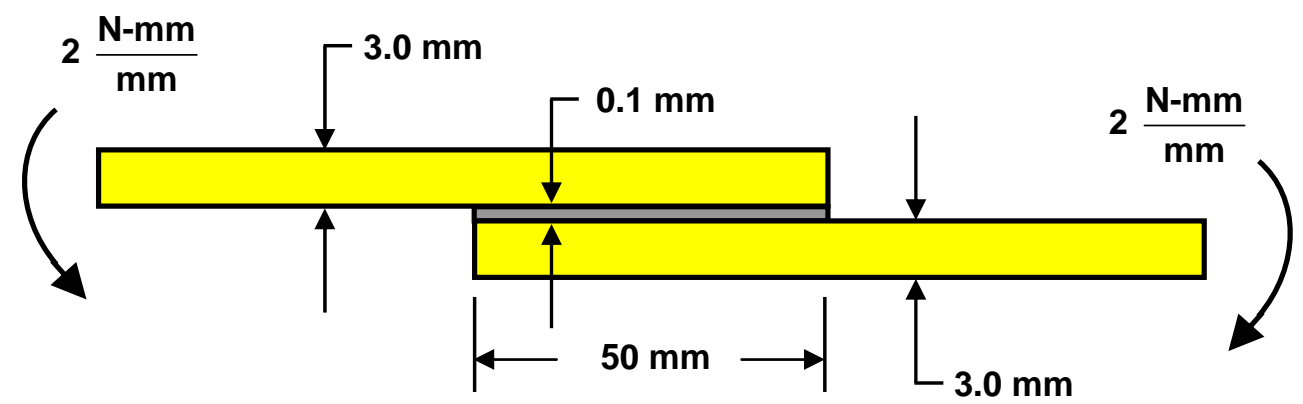

Figure 3. Single-lap joint geometry and loading (from Yang ${ }^{3}$ ) used for the ABAQUS analyses.

The shear and normal stress responses for the single-lap joint model from ABAQUS are presented in Figures 9 and 10 along with the results determined using ABJO. Analyses were conducted using both the coarse and fine mesh models in ABAQUS, and those results demonstrated that the solution had converged by providing less than a $5 \%$ difference for the peak stress values. However, the solutions provided almost identical results for the adhesive shear and normal stress responses, and are therefore not presented in the present paper. The fine mesh model was used to conduct the analyses used to compare results with ABJO in Figures 9 and 10. Comparison of the adhesive stress responses between ABAQUS and ABJO again provided close correlation between the two analysis methods; however, a greater difference in the adhesive stress responses from $\mathrm{ABJO}$ and $\mathrm{ABAQUS}$ were determined compared to the results by Yang. ${ }^{3}$ In the case of the adhesive shear stress response, the ABJO results had very little variation from the ABAQUS results except near the end of the overlap region. The largest difference occurred at the ends of the joint overlap 
region, where the peak shear stress for the ABAQUS fine mesh model is $0.132 \mathrm{~N} / \mathrm{mm}^{2}$ and the peak shear stress for the ABJO analysis is $0.15 \mathrm{~N} / \mathrm{mm}^{2}$. The $13 \%$ difference between the two peak adhesive shear stresses is most likely due to the higher predicted shear stress at the 0.2 and 0.8 joint station locations for the ABAQUS model, which would tend to reduce the adhesive shear stress peaking near the joint overlap ends. The adhesive normal stress response for the two analysis methods displayed a much larger variation than in the case of the shear stress response. A larger variation between the two adhesive normal stress responses should not be surprising as solutions are quite sensitive at the overlap ends due to the singularity from the geometric discontinuity at the free edge. The peak adhesive normal stress determined from ABJO is $0.321 \mathrm{~N} / \mathrm{mm}^{2}$ while the peak obtained from ABAQUS is $0.51 \mathrm{~N} / \mathrm{mm}^{2}$. Thus, a $38 \%$ difference was determined between the peak adhesive normal stresses for ABJO and ABAQUS. Also, a large difference between the two adhesive normal stress responses was determined just inboard of the joint ends, where secondary peaks are located at the 0.1 and 0.9 joint station locations. The compressive normal stress produced at the 0.1 joint station location and the tensile normal stress at the 0.9 joint station is typically due to large bending gradients at the ends of the joints. As the bending near the ends of the overlap region increases, so do the secondary peaks at the 0.1 and 0.9 joint stations. The reduction in the secondary peaks influences the peak at the joint overlap ends, causing the adhesive normal stress to rise at the ends of the overlap. A possible explanation for the significant difference in the adhesive normal stress responses as well as the smaller difference in the adhesive shear stress responses between the ABJO and ABAQUS results is that the ABAQUS models may be overly stiff due to the use of fully integrated, four-node quadrilateral elements. The increased model stiffness may be limiting the bending response of the models, and thus increasing the peak normal stress at the overlap ends. However, for the adhesive shear stress response, the stiffer bending response of the ABAQUS models would tend to reduce the load transition from one adherend to the other at the ends of the joint and force more load to transfer towards the center of the overlap region. Thus, the artificially stiff bending of the ABAQUS model would affect the adhesive shear stress by reducing the peak shear stress value and increasing the shear stress further from the joint overlap ends as demonstrated by the adhesive shear stress responses for the ABAQUS model compared to the ABJO solution in Figure 9.

\section{Conclusions}

The semi-analytical analysis method ABJO was verified using a single-lap joint subjected to pure bending from the literature. Results from ABJO showed close agreement with both the results determined by Yang and linear static finite element analyses conducted using the commercial software ABAQUS. The adhesive shear stress response for ABJO and the ABAQUS models had close correlation for the majority of the overlap length with the largest difference in the adhesive shear stress results occurring at the ends of the joint overlap region. The peak shear and normal stresses occurred, as expected, at the ends of the joint overlap regions for both the ABJO and ABAQUS analyses. The peak shear stress for the ABAQUS fine mesh model was $0.132 \mathrm{~N} / \mathrm{mm}^{2}$ and the peak shear stress for the ABJO analysis was $0.15 \mathrm{~N} / \mathrm{mm}^{2}$, while the peak adhesive normal stress determined from ABJO was $0.321 \mathrm{~N} / \mathrm{mm}^{2}$ and the peak obtained from ABAQUS was $0.51 \mathrm{~N} / \mathrm{mm}^{2}$. These values for the peak adhesive shear and normal stress values for ABJO and ABAQUS represented a difference of $13 \%$ and $38 \%$, respectively. Thus, the semi-analytical methodology was successfully verified using the ABAQUS finite element software and a single-lap joint configuration subjected to pure bending. While further validation using experimental data is warranted, the ABJO analysis method should provide the design engineer an efficient analysis tool for evaluating adhesively bonded joints. 


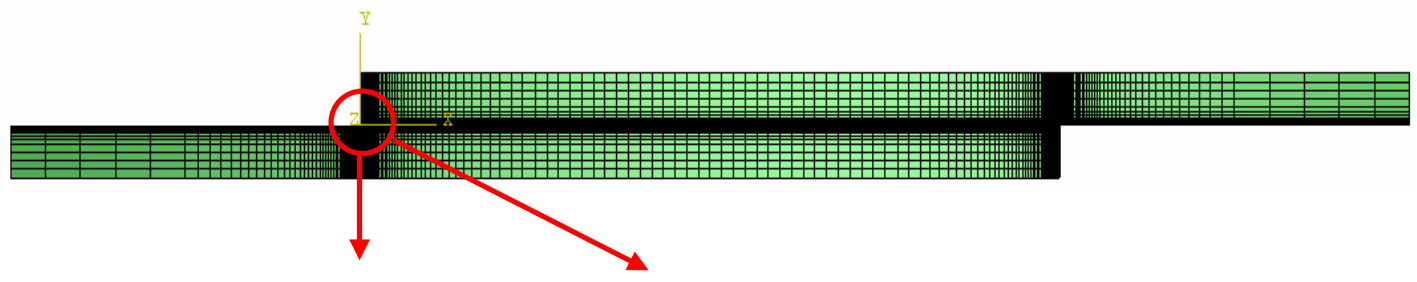

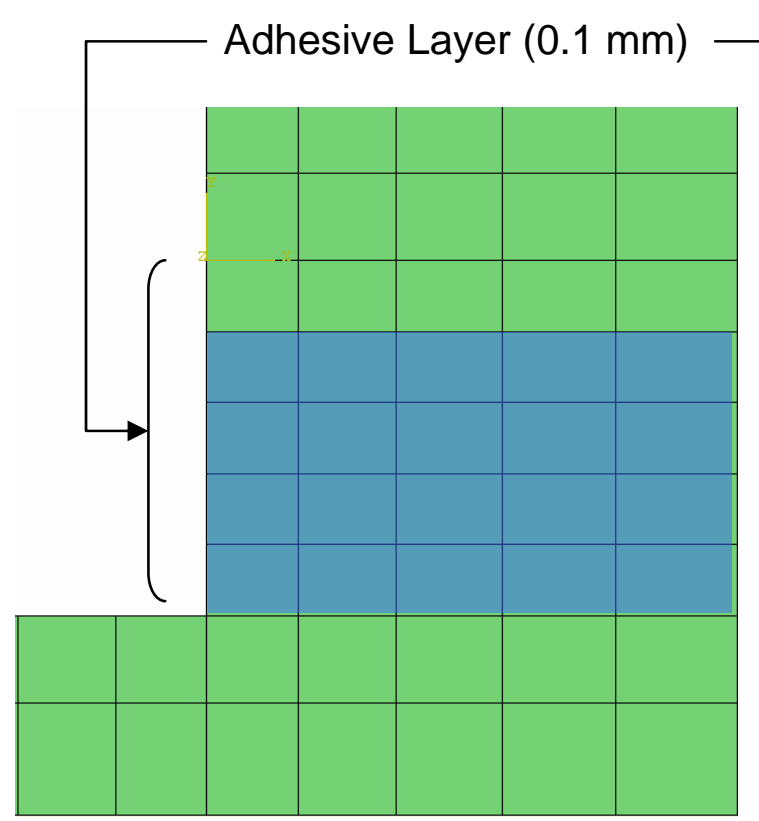

Coarse Model

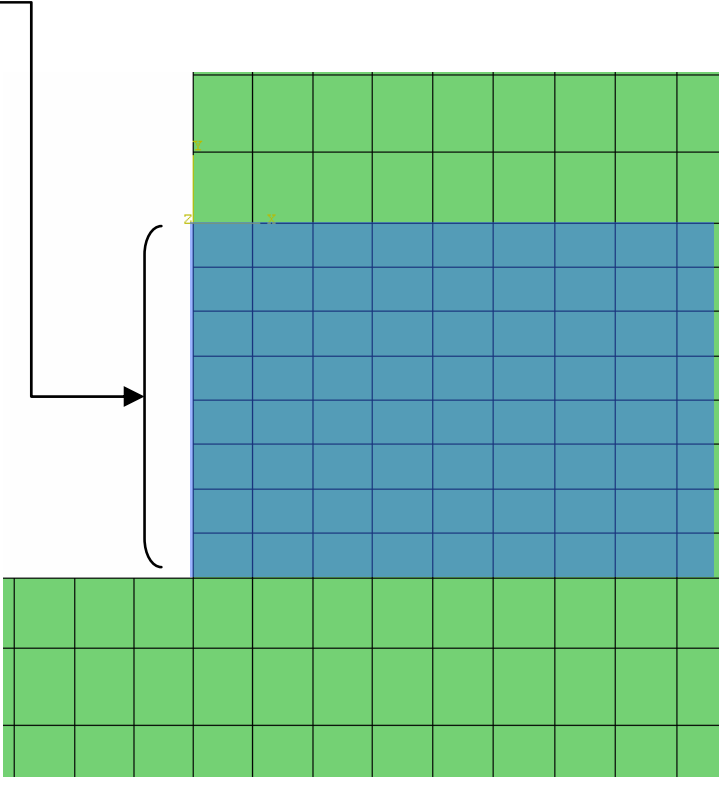

Fine Model

Figure 4. Mesh density for the single-lap joint models and the local mesh density at the ends of the bonded overlap region for both the coarse and fine models.

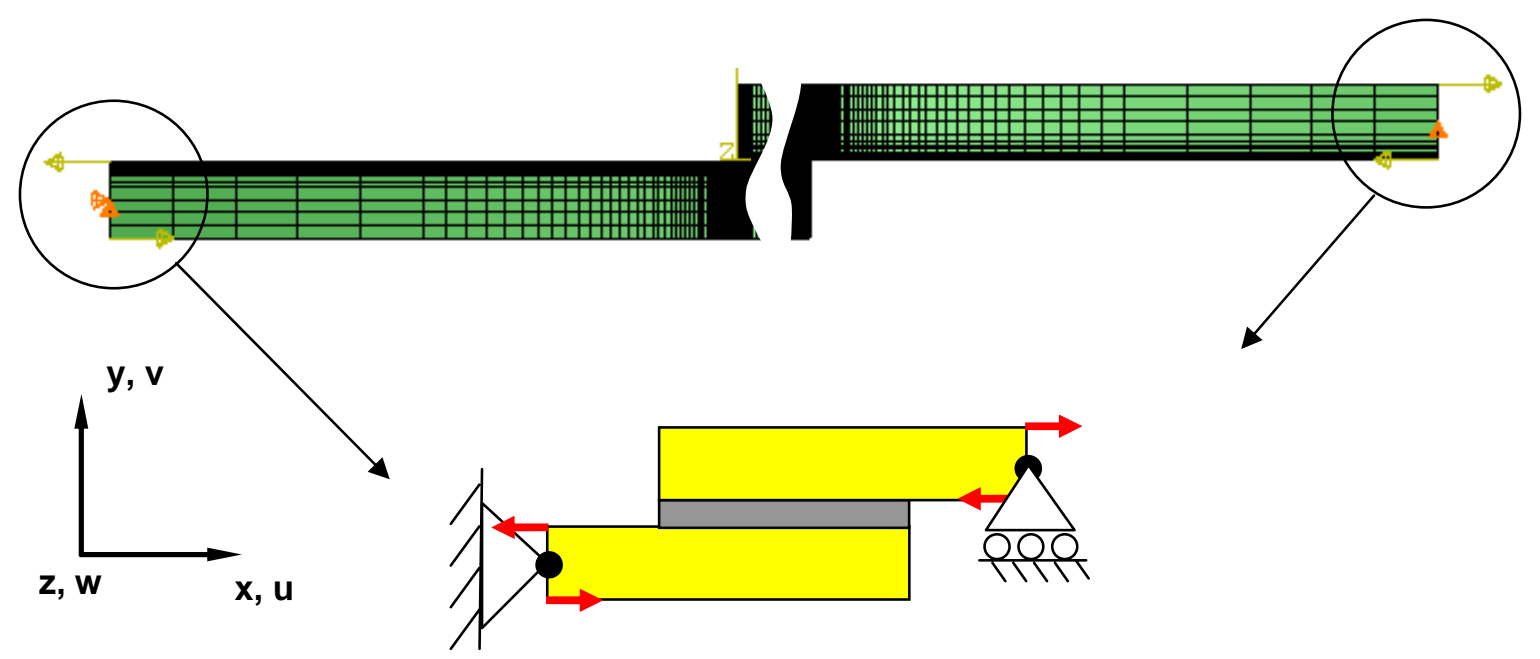

Figure 5. Boundary conditions for the single-lap joint ABAQUS models, where the lower adherend is pinned and the upper adherend is permitted two degrees of freedom. 


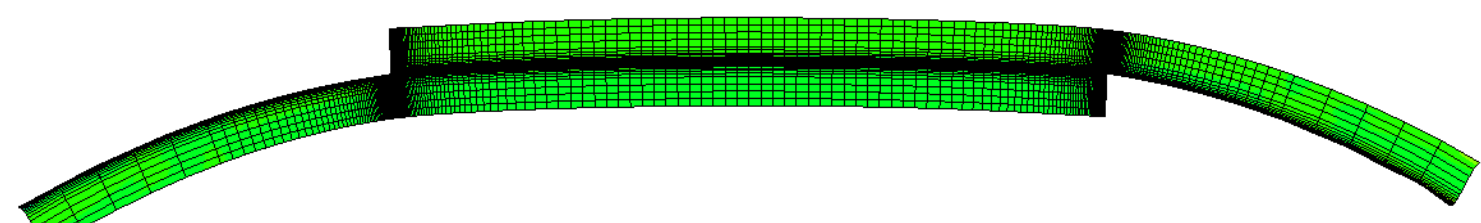

a) Global deformed shape

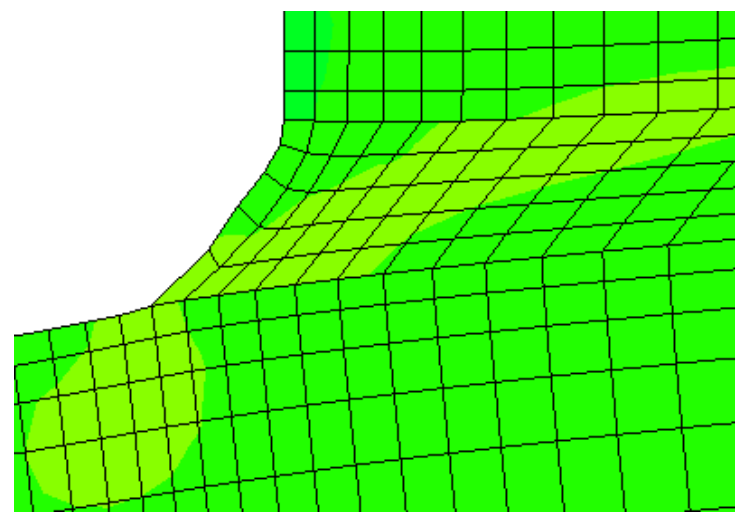

b) Coarse mesh

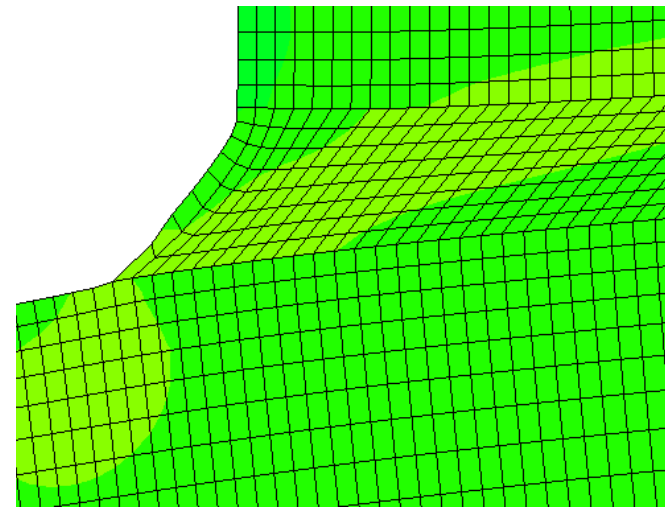

c) Fine mesh

Figure 6. a) Global deformed shape of the single-lap joint model, and the local deformed shape near the end of the adhesive overlap region for the b) coarse and c) fine mesh models.

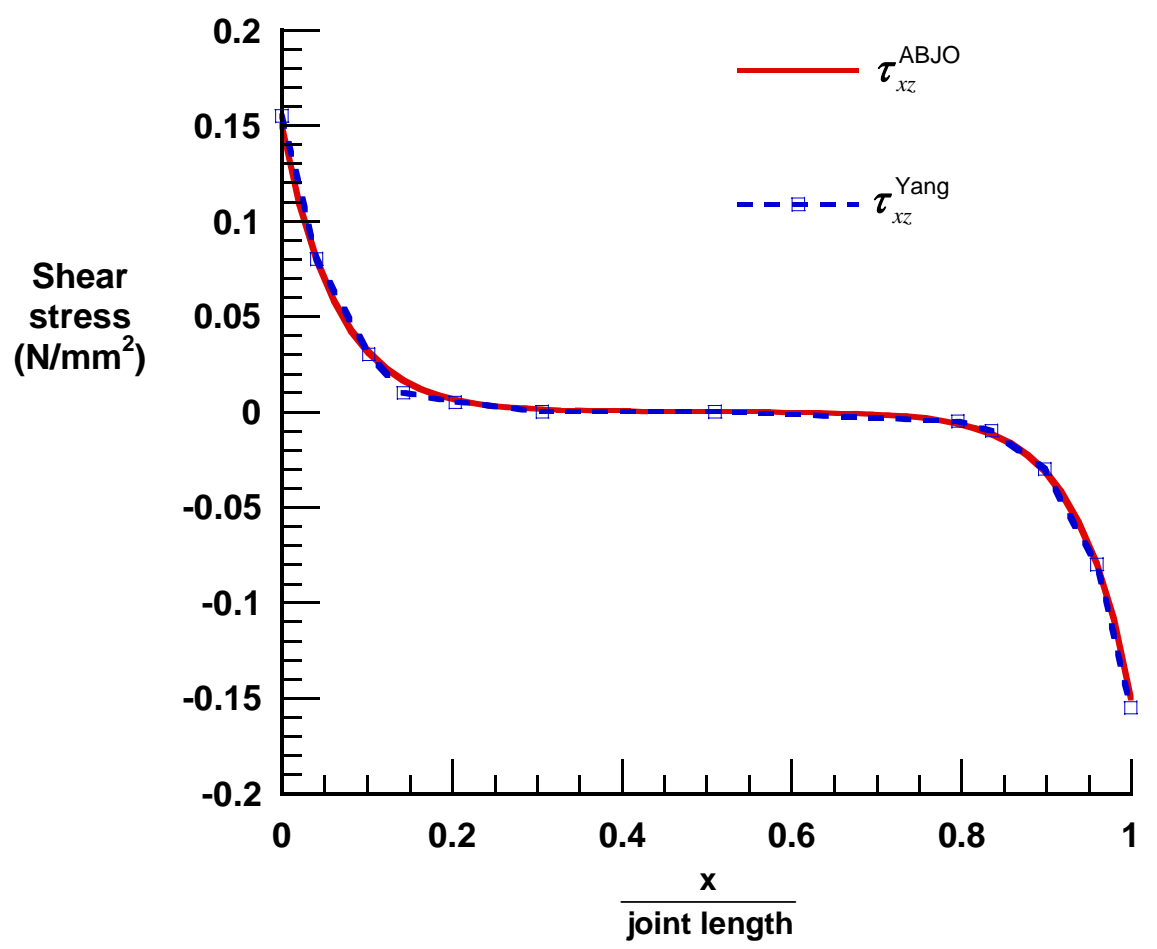

Figure 7. Adhesive shear stress response as a function of the nondimesionalized overlap length from ABJO and Yang $^{3}$ for the case of a single-lap joint subjected to a $2 \mathrm{~N}-\mathrm{mm} / \mathrm{mm}$ bending load. 


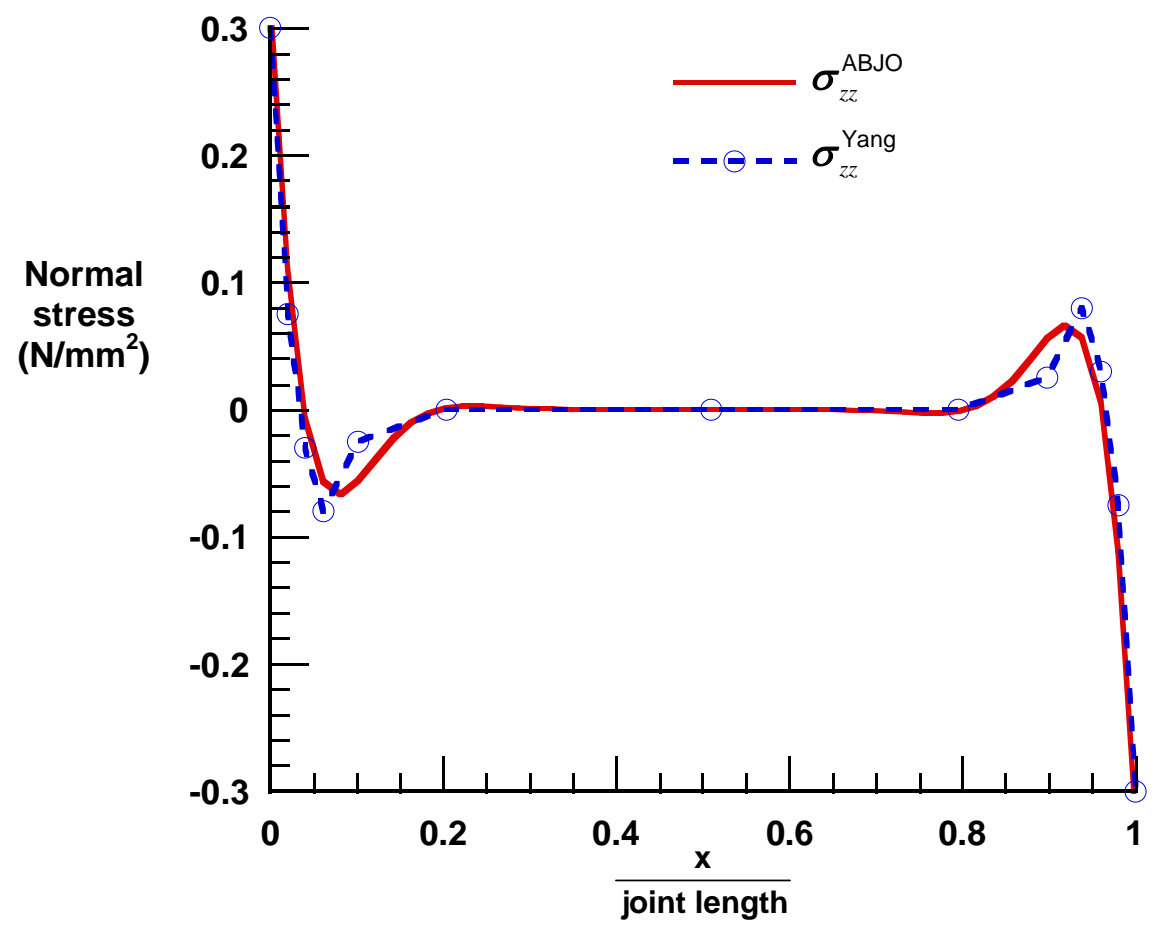

Figure 8. Adhesive normal stress response as a function of the nondimesionalized overlap length from ABJO and Yang ${ }^{3}$ for the case of a single-lap joint subjected to a $2 \mathrm{~N}-\mathrm{mm} / \mathrm{mm}$ bending load.

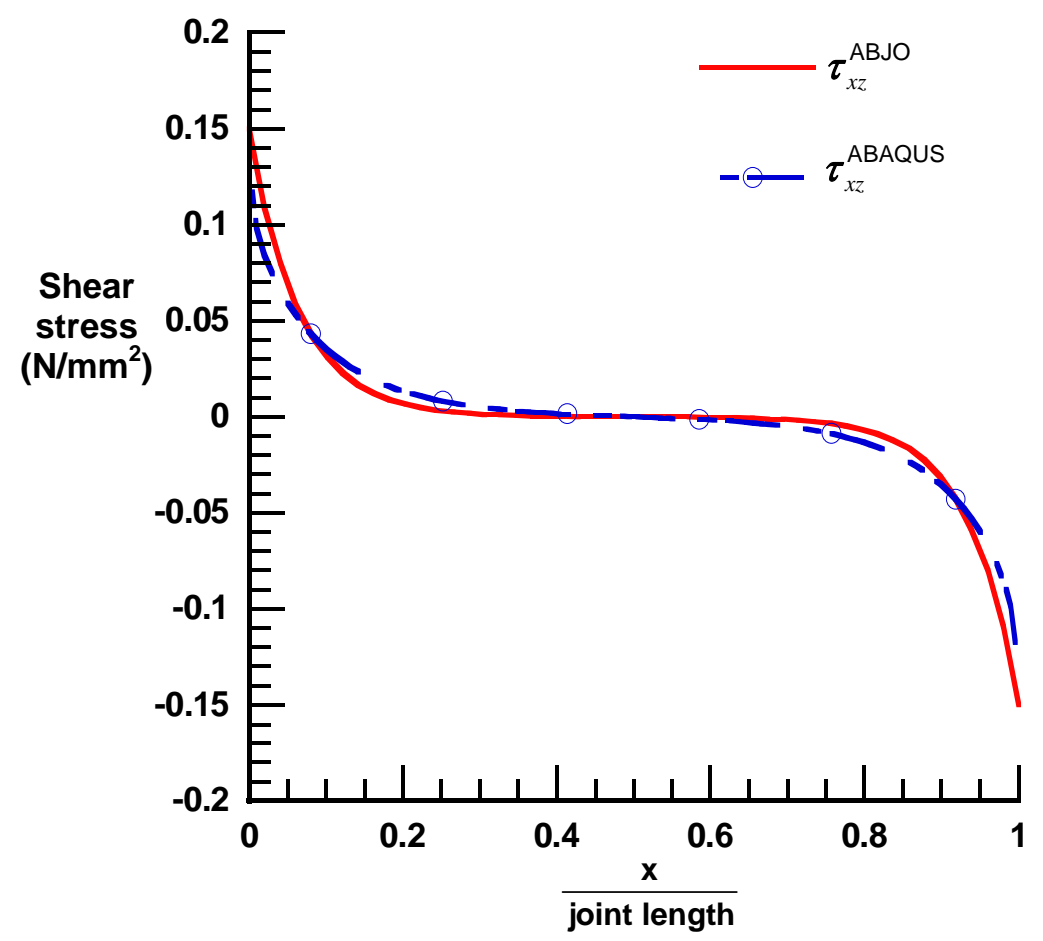

Figure 9. Adhesive shear stress response as a function of the nondimesionalized overlap length from ABJO and ABAQUS for the case of a single-lap joint subjected to a $2 \mathrm{~N}-\mathrm{mm} / \mathrm{mm}$ bending load. 


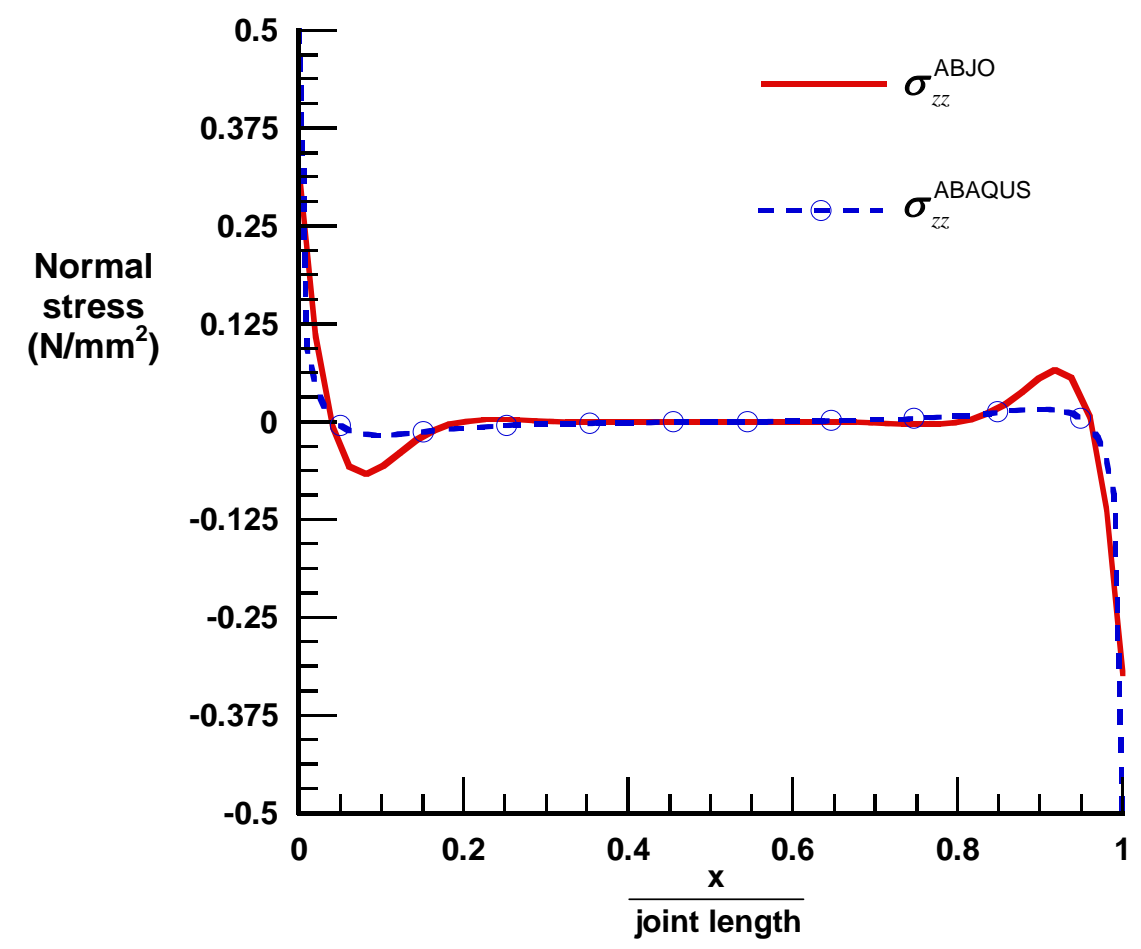

Figure 10. Adhesive normal stress response as a function of the nondimesionalized overlap length from ABJO and ABAQUS for the case of a single-lap joint subjected to a $2 \mathrm{~N}-\mathrm{mm} / \mathrm{mm}$ bending load.

\section{References}

${ }^{1}$ Smeltzer, S.S., “An Inelastic Analysis Methodology for Bonded Joints with Shear Deformable, Anisotropic Adherends,” Ph.D. Dissertation, North Carolina State University, Raleigh, NC, 2003.

${ }^{2}$ Smeltzer, S.S. and Klang, E.C., "Analysis method for inelastic, adhesively bonded joints with anisotropic adherends," Proceedings of the $18^{\text {th }}$ American Society for Composites Conference, Orlando, Florida, October 19-22, 2003.

${ }^{3}$ Yang, C., Huang, H., Tomblin, J.S., and Sun, W., "Elastic-Plastic Model of Adhesive-Bonded Single-Lap Composite Joints,” J. of Composite Materials, Vol. 38, No. 4, 2004, pp. 293-309.

${ }^{4}$ Mortensen, F. and Thomsen, O.T., "Analysis of adhesive bonded joints: a unified approach,” Composites Science and Technology, Vol. 62, No. 7/8, 2002, pp. 1011-1031.

${ }^{5}$ Oterkus, E., Barut, A., Madenci, E., Smeltzer, S.S., and Ambur, D.R., "Nonlinear analysis of bonded composite single-lap joints,” AIAA-2004-1560, Proceedings of the 45th AIAA/ASME/AHS Structures, Structural Dynamics, and Materials Conference, Palm Springs, CA, April 19-22, 2004.

${ }^{6}$ Oterkus, E., Madenci, E., Smeltzer, S.S., and Ambur, D.R., "Nonlinear analysis of bonded composite tubular lap joints," Proceedings of the 46th AIAA/ASME/AHS Structures, Structural Dynamics, and Materials Conference, Austin, TX, April 17-21, 2005.

${ }^{7}$ ABAQUS. User’s manuals, version 6.5. ABAQUS, Inc.; 2004. 